\title{
Photon-number squeezing in nano and microlasers
}

Mark Anthony Carroll, ${ }^{1}$ Giampaolo D'Alessandro, ${ }^{2}$ Gian Luca Lippi, ${ }^{3}$ Gian-Luca Oppo, ${ }^{1}$ and Francesco Papoff ${ }^{1}$ a) ${ }^{1)}$ Department of Physics, University of Strathclyde, 107 Rottenrow, Glasgow G4 ONG,

United Kingdom.

${ }^{2)}$ School of Mathematical Sciences, University of Southampton, Southampton SO17 1BJ,

United Kingdom

${ }^{3)}$ Université Côte d'Azur, UMR 7710 CNRS, Institut de Physique de Nice, 1361 Route des Lucioles, 06560 Valbonne,

France

(Dated: 23 August 2021)

Based on theoretical predictions on the appearance of antibunching before the laser threshold at the nano- and microscale, we analyze the amount of photon-number squeezing naturally produced in the laser emission. Up to $3 \mathrm{~dB}$ photon number noise reduction is obtained in comparison to the coherent emission, with output power estimated as a fraction of a $\mathrm{nW}$ and with negligible effects due to pump fluctuations. The scheme requires a high Q cavity and holds promise for the construction of a simple and effective photon-number squeezed source.

Measurement systems are eventually limited in their performance by the presence of noise, whose reduction benefits metrology, quantum imaging, optical communications and measurements, gravitational wave detection, quantum information, etc. ${ }^{1-5}$. The strongest efforts to reduce photon number noise through light squeezing have been concentrated onto the single emitter regime, with a record fluctuation reduction of about $3 \mathrm{~dB}$ in single Quantum Dot resonance fluorescent emission ${ }^{6}$.

Photon number squeezing was investigated early on ${ }^{7,8}$ for its relative ease of implementation and for its compatibility with a large photon flux. Numerous implementations, based on direct photon number squeezing ${ }^{9}$ on pulsed nonlinear schemes with up to $10^{10}$ photons ${ }^{10,11}$, or superadian effects ${ }^{12,13}$ have been predicted but they often produce only a low amount of squeezing (fraction of a dB). More recently, squeezing has been obtained from a set up based on cold atomic samples but at the cost of a complex realization ${ }^{14}$

Clustering of emitters or entangled photon ensembles represent another way of producing multiphoton squeezed states for sophisticated computing or cryptographic applications ${ }^{15-17}$. Polaritons in the strong coupling regimes have produced a good amount of squeezing and hold promise as sources of light for continuous variable quantum information encoding and cryptography ${ }^{18}$. Advanced nonlinear resonator concepts ${ }^{19-21}$ now enable the realisation of interesting and flexible schemes which could find use in integrated devices. However, all previous schemes require rather complex experimental setups and recently interest is developing for sources where intensity noise can be reduced with simpler schemes $^{22,23}$ (and references therein). The device which we propose provides below-threshold squeezing and is based on a nanostructure. Thus, in spite of an output power lower than what has been previously obtained, it offers several advantages: small footprint and thermal load, thus enabling on-chip integration; avoidance of multimode anticorrelations which, while providing squeezed light ${ }^{24}$, render the photon stream not usable for numerous applications ${ }^{25}$; avoidance of feed-

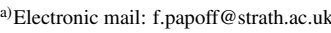

back schemes ${ }^{26-28}$ and of cooling requirements ${ }^{29,30}$. The intrinsic integrability of the proposed source into optical chips offsets its intrinsic low photon flux, since virtually all photons can be used in a guided structure. In addition, giant nonlinearities emerging in nanostructures ${ }^{31}$, metamaterials ${ }^{32}$, plasmonics ${ }^{33}$ and quantum interference ${ }^{34}$ hold strong promise for the exploitation of weak signals in integrated structures.

In line with these developments, the recent quantum-dot based Coherent-Incoherent model (CIM), where the coherent and incoherent field components are independently described $^{35}$, predicts from first principles the existence of a prethreshold regime where photon antibunching, thus squeezing $^{36}$, is naturally observed. The CIM model has been derived under the experimentally verified conditions ${ }^{37}$ that the decay rate of the material polarization is much larger than that of the photons in the cavity. In this case it is well known ${ }^{38}$ that the emitters are independent from each other and that correlations between emitters are negligible even close to laser threshold. The pump range in which antibunching appears is broad enough to promise experimental accessibility and the resulting degree of squeezing sizeable enough to warrant consideration. Antibunching has been experimentally observed and modeled ${ }^{39}$ for a low number of emitters. In this letter we predict that squeezing with large photon number occurs at extremely low power supply for nano and micro laser with emitter numbers up to $10^{3}$ in the anti-bunching region ${ }^{35}$. The advantage of this prediction is that the photon antibunched behaviour appears to naturally precede lasing, in a cw regime of operation, without the need for any special experimental arrangements and promises to provide photon fluxes comparable or larger than those of more complex, pulsed techniques.

In order to introduce the ideas, we generalize the relationship between average and variance in a Poisson process by introducing a sub-Poissonian coefficient $a$ :

$$
\left\langle\Delta n^{2}\right\rangle=a\langle n\rangle
$$

with $n$ photon number, \langle\rangle temporal average and $\left\langle\Delta n^{2}\right\rangle$ corresponding to the variance, where a subpossonian statistics, i.e. squeezed emission, emerges for $a<1$. In order to connect squeezing to antibunching we introduce this modified distribution into the definition of the zero-dealy second-order auto- 


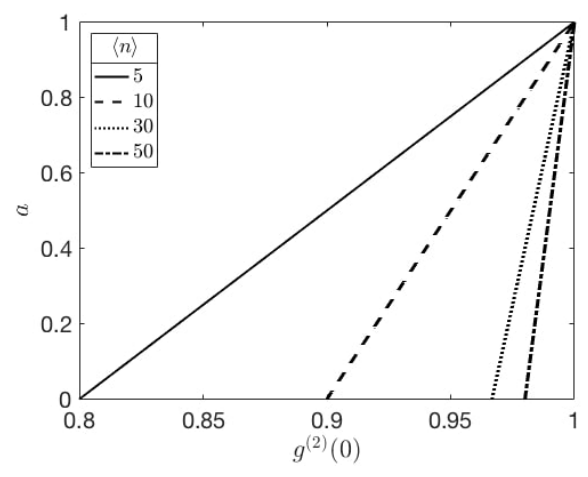

FIG. 1. Subpoissonian coefficient $a$ (defined in eq. (1)) as a function of the value of $g^{(2)}(0)$ for different values of the average photon number $\langle n\rangle$.

correlation function

$$
g^{(2)}(0)=\frac{\left\langle n^{2}\right\rangle-\langle n\rangle}{\langle n\rangle^{2}}=1-\frac{1-a}{\langle n\rangle} .
$$

Photons are antibunched when $g^{(2)}(0)<1$, so that $g^{(2)}(0)$ is a standard measure of the degree of antibunching. Solving equation (2) for $a$, we notice that it linearly depends on the average photon number $\langle n\rangle$. As the photon number grows, the apparent degree of antibunching is reduced for a same reduction in the relative fluctuation eq. (1) as illustrated in Fig. 1.

Thus, even though the antibunching may appear to be small, it is possible to maintain a good amount of photon number noise reduction, thus squeezing since $g^{(2)}(0)<1$ (see Refs. ${ }^{40-42}$ and references therein), in the photon number for macroscopic signals. These qualitative considerations point to a potentia interest even in a moderate amount of antibunching emerging from the model ${ }^{35}$

The main interest of the scheme is its intrinsic simplicity and the potential for effective photon number squeezing with considerable large photon numbers. The fact that a belowthreshold nanolaser may spontaneously emit photon streams in a more ordered fashion than an equivalent coherent source ${ }^{43}$ hints to potential interest for a variety of applications. Indeed, in this configuration there is no need to destroy phase coherence to reduce photon number noise, contrary to what is required of above-threshold lasers.

We test the idea by using a quantum model ${ }^{35}$, derived using the cluster expansion technique ${ }^{44,45}$, that includes both coherent and incoherent field components; previous cluster expansion based models neglect the expectation values of the coherent variables ${ }^{46-48}$.Its key feature resides in the univocal determination of a lasing threshold irrespective of cavity characteristics (even for $\beta=1$, the fraction of spontaneous emission coupled into the lasing mode), thus establishing a clear boundary between the squeezed and coherent emission regime. The medium is assumed to be an ensemble of iden-

\begin{tabular}{llr}
\hline \hline Parameter & Value & Description \\
\hline$g$ & $7 \times 10^{10} \mathrm{~s}^{-1}$ & Light-matter coupling strength \\
$\gamma_{c}$ & $7 \times 10^{9} \mathrm{~s}^{-1}$ & Cavity decay rate \\
$\gamma$ & $10^{13} \mathrm{~s}^{-1}$ & Dephasing rate of active medium \\
$\gamma_{n r}$ & $10^{9} \mathrm{~s}^{-1}$ & nonradiative decay rate \\
$\gamma_{n l}$ & $1.4 \times 10^{12} \mathrm{~s}^{-1}$ & Decay rate into the nonlasing modes \\
$\beta$ & $7 \times 10^{-4}$ & Spontaneous emission factor \\
\hline \hline
\end{tabular}

TABLE I. Parameters that appear in the model used to compute $g^{(2)}(0)$, equation (3). The values in this table are used in all figures unless stated otherwise.

tical but independent two-level emitters coupled with a single cavity lasing mode. We remember that the rapid polarisation dephasing permits one to omit inter-emitter correlations ${ }^{38,46}$. The incoherent dynamical variables are the population density of the excited state, $\left\langle c^{\dagger} c\right\rangle$; the photon assisted polarisation of the medium, $\delta\left\langle b c^{\dagger} v\right\rangle$; and the number of photons, $\delta\left\langle b^{\dagger} b\right\rangle$. These are coupled to the coherent variables, i.e. the electric field amplitude $\langle b\rangle$ and the classical polarisation $\left\langle v^{\dagger} c\right\rangle$.

The lasing threshold exists only if the total number $N$ of emitters in the cavity is larger than a critical value $N_{c}$. If this condition is fulfilled, then at sufficiently large pump values a stable coherent field begins to grow inside the cavity. This has also been confirmed in a standard rate equation model that includes stochastic noise terms ${ }^{49}$ as well as experimentally ${ }^{48}$.

Using this model we have computed the intra-cavity value

$$
g^{(2)}(0)=\frac{\left\langle b^{\dagger} b^{\dagger} b b\right\rangle}{\left\langle b^{\dagger} b\right\rangle^{2}}
$$

expressed in terms of field creation and destruction operators, as a function of the model parameters summarised in table I. The important parameters that control and characterise squeezing are the pump parameter per emitter, $r$, the number of emitters, $N$, the cavity decay rate, $\gamma_{c}$ and the light-matter coupling strength, $g$. The remaining system parameters are the decay rate of the medium, $\gamma$; the non-radiative decay rate, $\gamma_{n r}$; and the decay rate into non-lasing modes, $\gamma_{n l}$. The latter controls $\beta$, where the ultimate limit of $\beta=1$ corresponds to $\gamma_{n l}=0$.

From here on, we concentrate on parameter values which match a microlaser, simply because we aim at obtaining macroscopic amounts of output power. The findings, however, generally hold for all devices down to the extreme nanoscale, with features which only change quantitatively. In Fig. 2 we plot the steady state values of $g^{(2)}(0)$ as a function of the pump for two different lasing devices. As the pump increases the two photon statistics (depending on the device) enter three different regimes: from super-Poissonian (thermal light) to subPoissonian (squeezed light), and, then, to Poissonian (coherent light) - in the good cavity limit ( $\gamma_{c}<g$, solid line); when cavity losses are equal to the coupling strength (dashed line) the antibunching becomes negligibly small and disappears entirely in the bad cavity regime $\left(\gamma_{c}>g\right.$, not plotted). The antibunching region shows a sensitive dependence on the number of emitters as there is only a small window of pump values 


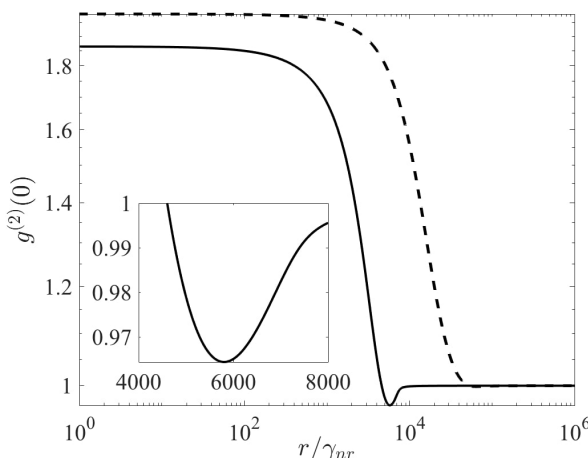

FIG. 2. The intensity autocorrelation function versus pump $r$ for different paired values of the cavity losses $\gamma_{c}$ and number of emitters $N$ above the critical number $N_{c}$ required for lasing. In order to meet the condition for lasing, as the cavity losses increase so to must the number of emitters, therefore $N=\{21,150\}$ and $\gamma_{c}=\left\{7 \times 10^{9} \mathrm{~s}^{-1}, 7 \times 10^{10} \mathrm{~s}^{-1}\right\}$ for the solid and dashed curves respectively.

for which squeezing can occur and this becomes narrower as the number of intra-cavity emitters increases ${ }^{35}$. As we later show, this constraint does not strongly affect the scheme's implementability.

Concentrating on $\gamma_{c}<g$, we remark that while squeezing is possible with a very small number of emitters, provided the coupling $g$ is sufficiently strong, a substantial amount of power is obtained only from devices which are capable of passing the laser threshold ${ }^{35}$, i.e. with $N>N_{c}$. We thus concentrate on this regime keeping the remaining values of table I fixed, as their influence on the final result is only minor.

To estimate the photon number noise reduction we directly compute the relative fluctuation of the photon number,

$$
\frac{\langle\Delta n\rangle}{\langle n\rangle}=\frac{\sqrt{\left\langle b^{\dagger} b b^{\dagger} b\right\rangle-\left\langle b^{\dagger} b\right\rangle^{2}}}{\left\langle b^{\dagger} b\right\rangle} .
$$

which can be transformed into an expression containing $g^{(2)}(0)$ by the normal-ordering ${ }^{45}$ of $\left\langle b^{\dagger} b b^{\dagger} b\right\rangle$ :

$$
\frac{\langle\Delta n\rangle}{\langle n\rangle}=\sqrt{\left\langle b^{\dagger} b\right\rangle^{-1}+g^{(2)}(0)-1} \text {. }
$$

As expected, noise reduction appears for $g^{(2)}(0)<1$. It is also important to note that as $N$ increases $g^{(2)}(0)$ approaches 1 and we recover the relative fluctuations of the classical limit, i.e. $1 / \sqrt{n}$.

However, the presence of antibunching ensures a more regular temporal distribution of photons than the random occurrences of coherent emission $^{43}$, thus squeezing, which we quantify by defining an attenuation coefficient

$$
A_{\mathrm{dB}}=20 \log _{10}\left(\frac{\langle\Delta n\rangle}{\langle n\rangle}\right)
$$

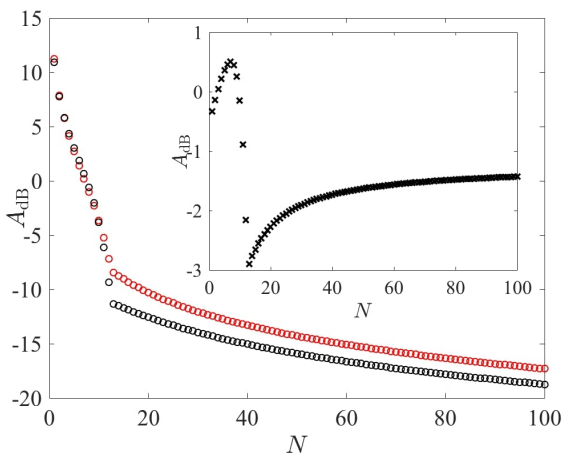

FIG. 3. Squeezing as a function of $N$. The black curve is obtained from equation (6) at the minimum of $g^{(2)}(0)$ calculated from the CIM, whereas the red curve shows squeezing for a coherent field. The inset shows how much squeezing is gained compared to a coherent field with the same photon number.

and comparing its value ${ }^{50}$ to the one which characterizes coherent emission $\left(g^{(2)}(0)=1\right)$.

We first consider the case of an ideal pump with no fluctuations and evaluate the attenuation at the minimum of $g^{(2)}(0)$, i.e., where squeezing is greatest. Fig. 3 compares the noise attenuation as a function of the emitter number (black circles) to the reference coherent field emission (red circles) obtained by imposing $g^{(2)}(0)=1$ in eq. (5). The inset shows the difference between the two. The best squeezing, $-3 \mathrm{~dB}$, for $N=13$, just below the critical number of emitters required for lasing, $N=15$, for the parameters used in Fig. 3. We see that even with 100 emitters there is still approximately $1.5 \mathrm{~dB}$ of squeezing. This is obtained with standard laser parameter values. With some technological efforts in the realization of dedicated devices and some optimization better results can be obtained.

The case just considered neglects pump fluctuations. However, incorporating them is of vital importance if we are to understand the viability of potential devices. Pump stabilization has been one of the first means of obtaining squeezing ${ }^{51,52}$ and technology is capable of achieving stability values well below $1 \%$ for the currents needed to pump a small device. We therefore consider a maximum amplitude fluctuation in the pump by $\pm 1 \%$ and observe its influence on the photon number noise reduction.

Fig. 4 shows the absolute noise reduction (as in the main panel of Fig. 3) for the below-threshold laser (orange shaded area) and for a coherent signal (green shaded area). The lower curve delimiting the region corresponds, for both cases, to a positive $1 \%$ fluctuation - enhancing the amount of power while the upper one represents the boundary set by a negative $1 \%$ fluctuation. In either case, the spread increases from something extremely small at when the number of emitters is at the minimum value, $N=15$, to a sizeable fluctuation for $N=100$. The fact that the antibunched curves lie below the 

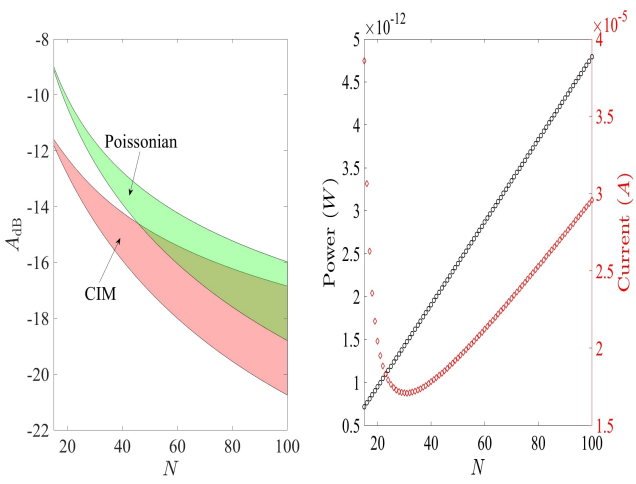

FIG. 4. Left panel: Spread in squeezing of the antibunching and coherent sources in Fig. 3 due to $\pm 1 \%$ fluctuations in the pump as a function of $N$ (taken above $N_{c}$ ). Right panel: Output power (black) and pump current (red) as a function of $N>N_{c}$. All points are evaluated at the minimum of $g^{(2)}(0)$.

coherent ones signal the fact that a large part of the contribution to the fluctuation originates from the change in photon number, rather than in a modification in the amount of antibunching (cf. inset in Fig. 3). Only the differentially larger growth of the fluctuation in the below-threshold laser (upper bound of the fluctuation, corresponding to the steeper part of the antibunching curve - inset of Fig. 3 ) is to be attributed to come to a change in the amount of antibunching. Since we have considered a rather large pump fluctuation, compared to what is technologically feasible, and the laser injection current can be controlled to a much better degree, it is reasonable to consider the influence of pump noise negligible.

The right panel of Fig. 4 displays the absolute amount of current needed to pump the laser at the optimum antibunching value as a function of the emitter number. The injected current is is, at $N=15$, about $40 \mu \mathrm{A}$ (red symbols) and, as already mentioned, can be easily stabilized to better than $1 \%$. The accompanying outcoupled output power (black circles computed on the basis of the intracavity photon number, cavity losses and photon energy at $\lambda=1 \mu \mathrm{m}$ ) is about $0.7 \mathrm{pW}$ (i.e., $\approx 10^{7}$ photons) around the intracavity $-3 \mathrm{~dB}$ squeezing level, and grows to $\approx 5 \mathrm{pW}$ for 100 emitters (and $\approx 1.5 \mathrm{~dB}$ squeezing). Given that better pump stabilization is achievable, we have checked the results with pump fluctuations of the order of $0.1 \%$. The stability is greatly improved and the output power can grow to $50 \mathrm{pW}$ with 1000 emitters with $\approx 1.25 \mathrm{~dB}$ squeezing still gained compared to a coherent source .

It is important to remark that the conditions we have examined here correspond to a "good cavity" device $\left(Q \approx 2 \times 10^{4}\right.$, following the definition of Ref. 48), as observed in Ref. 39 with a number of quantum dots consistent with the numbers used in this work. Larger photon fluxes can be obtained by increasing the cavity losses (thus decreasing Q) provided the coupling factor $g$ is correspondingly increased. Since we have used a standard value for $g$, routinely achieved in technological realizations, there is some margin for improvement there. It is likely that a careful choice of parameters and technological efforts may improve by one order of magnitude the power expected at the output of the device.

In summary, a recent model, which treats the incoherent and coherent parts of the field for lasers to describe the emission of Quantum-Dot based small-scale (nano- and micro-) lasers, predicts the appearance of photon emission with antibunched statistics before the lasing threshold ${ }^{35}$. Following the lead that this more regular photon emission produces reduced fluctuations, we have analyzed its properties with realistic physical construction parameters of a microlaser in the intent of obtaining a macroscopic output. The model predicts up to $3 \mathrm{~dB}$ photon-number squeezing with little influence of pump fluctuations, compared to the noise floor of an equivalent coherent field and output power approximately $0.7 \mathrm{pW}$. The squeezing is naturally produced by the physical interaction, without the need for any external action, since - contrary to standard squeezing of coherent output - below threshold there is no macroscopic phase to be degraded. Developments of this investigation include its extension to nanolasers to explore the exploitation of the same phenomenon at low photon numbers.

The Strathclyde and UCA research is conducted within the context of the International Associated Laboratory "SelfOrganization in Lasers and Atoms and Coherence Effects"-LIA SOLACE. GLL is grateful to V. D'Auria for enlightening discussions and acknowledges support from the French government through its Investments for the Future programme under the Université Côte d'Azur UCA-JEDI project managed by the ANR.

The data that support the findings of this study are available from the corresponding author upon reasonable request.

${ }^{1}$ V. Giovannetti, S. Lloyd, and L. Maccone, Nature photonics 5, 222 (2011). ${ }^{2}$ Y. Yamamoto and H. Haus, Reviews of Modern Physics 58, 1001 (1986). ${ }^{3}$ C. M. Caves, Physical Review D 23, 1693 (1981).

${ }^{4}$ J. Aasi, J. Abadie, B. Abbott, R. Abbott, T. Abbott, M. Abernathy, C. Adams, T. Adams, P. Addesso, R. Adhikari, et al., Nature Photonics 7, 613 (2013).

${ }^{5}$ U. L. Andersen, G. Leuchs, and C. Silberhorn, Laser \& Photonics Reviews 4, 337 (2010).

${ }^{6}$ H. Wang, J. Qin, S. Chen, M.-C. Chen, X. You, X. Ding, Y.-H. Huo, Y. Yu, C. Schneider, S. Höfling, et al., Physical Review Letters 125, 153601 (2020).

${ }^{7}$ Y. Yamamoto, S. Machida, and O. Nilsson, Physical Review A 34, 4025 (1986)

${ }^{8}$ S. Machida and Y. Yamamoto, Physical review letters 60, 792 (1988).

${ }^{9}$ A. Heidmann, R. Horowicz, S. Reynaud, E. Giacobino, C. Fabre, and G. Camy, Physical review letters 59, 2555 (1987).

${ }^{10}$ D. Smithey, M. Beck, M. Belsley, and M. Raymer, Physical review letters 69, 2650 (1992).

${ }^{11}$ P. Sharapova, A. M. Pérez, O. V. Tikhonova, and M. V. Chekhova, Physical Review A 91, 043816 (2015).

${ }^{12}$ V. V. Temnov and U. Woggon, Optics express 17, 5774 (2009).

${ }^{13}$ D. Meiser and M. Holland, Physical Review A 81, 063827 (2010).

${ }^{14}$ J. G. Bohnet, Z. Chen, J. M. Weiner, D. Meiser, M. J. Holland, and J. K. Thompson, Nature 484, 78 (2012).

${ }^{15}$ O. Shcherbina, G. Shcherbina, M. Manceau, S. Vezzoli, L. Carbone, M. De Vittorio, A. Bramati, E. Giacobino, M. Chekhova, and G. Leuchs, Optics letters 39, 1791 (2014).

${ }^{16}$ G. Harder, T. J. Bartley, A. E. Lita, S. W. Nam, T. Gerrits, and C. Silberhorn, Physical review letters 116, 143601 (2016) 
${ }^{17}$ L. Qi, M. Manceau, A. Cavanna, F. Gumpert, L. Carbone, M. de Vittorio, A. Bramati, E. Giacobino, L. Lachman, R. Filip, et al., New Journal of Physics 20, 073013 (2018).

${ }^{18}$ T. Boulier, M. Bamba, A. Amo, C. Adrados, A. Lemaitre, E. Galopin, I. Sagnes, J. Bloch, C. Ciuti, E. Giacobino, et al., Nature communications

5,1 (2014).
${ }_{19}$ M.-A. Lemonde, N. Didier, and A. A. Clerk, Physical Review A 90, 063824 (2014).

${ }^{20}$ H. Flayac and V. Savona, Physical Review A 96, 053810 (2017)

${ }^{21}$ H. Snijders, J. Frey, J. Norman, H. Flayac, V. Savona, A. Gossard, J. Bowers, M. van Exter, D. Bouwmeester, and W. Löffler, Physical review letter 121, 043601 (2018).

${ }^{22}$ J. Mork and K. Yvind, Optica 7, 1641 (2020).

${ }^{23}$ A. Musiał, K. Żołnacz, N. Srocka, O. Kravets, J. Große, J. Olszewski, K. Poturaj, G. Wójcik, P. Mergo, K. Dybka, et al., Advanced Quantum Technologies 3, 2000018 (2020).

${ }^{24}$ S. Inoue, H. Ohzu, S. Machida, and Y. Yamamoto, Physical Review A 46 2757 (1992).

${ }^{25}$ F. Marin, A. Bramati, E. Giacobino, T.-C. Zhang, J.-P. Poizat, J.-F. Roch, and P. Grangier, Physical review letters 75, 4606 (1995).

${ }^{26} \mathrm{M}$. Freeman, H. Wang, D. G. Steel, R. Craig, and D. Scifres, Optics letters 18, 2141 (1993).

${ }^{27}$ H. Wang, M. J. Freeman, and D. G. Steel, Physical review letters 71, 395 (1993).

${ }^{28}$ J. Kitching, A. Yariv, and Y. Shevy, Physical review letters 74, 3372 (1995)

${ }^{29}$ W. Richardson, S. Machida, and Y. Yamamoto, Physical review letters 66 , 2867 (1991).

${ }^{30}$ M. Freeman, H. Wang, D. Steel, R. Craig, and D. Scifres, Optics letters 18 379 (1993).

${ }^{31}$ M. Ren, B. Jia, J.-Y. Ou, E. Plum, J. Zhang, K. F. MacDonald, A. E. Nikolaenko, J. Xu, M. Gu, and N. I. Zheludev, Advanced Materials 23, 5540 (2011).

${ }^{32}$ J.-Y. Ou, E. Plum, J. Zhang, and N. I. Zheludev, Advanced Materials 28 729 (2016).

${ }^{33}$ P. N. Melentiev, A. E. Afanasiev, A. A. Kuzin, A. S. Baturin, and V. I.
Balykin, Optics express 21, 13896 (2013)

${ }^{34}$ S. Houver, A. Lebreton, T. A. Pereira, G. Xu, R. Colombelli, I. Kundu, L. Li, E. H. Linfield, A. Davies, J. Mangeney, et al., Science advances $\mathbf{5}$, eaaw7554 (2019).

${ }^{35}$ M. A. Carroll, G. D'Alessandro, G. L. Lippi, G.-L. Oppo, and F. Papoff, Physical Review Letters 126, 063902 (2021)

${ }^{36} \mathrm{H}$. Carmichael, Physical review letters 55, 2790 (1985).

${ }^{37}$ M. Sugawara, K. Mukai, Y. Nakata, H. Ishikawa, and A. Sakamoto, Physical Review B 61, 7595 (2000).

${ }^{38}$ I. E. Protsenko, A. Uskov, E. C. André, J. Moerk, and M. Wubs, New Journal of Physics (2021)

${ }^{39}$ J. Wiersig, C. Gies, F. Jahnke, M. ABmann, T. Berstermann, M. Bayer,

C. Kistner, S. Reitzenstein, C. Schneider, S. Höfling, et al., Nature $\mathbf{4 6 0}$ 245 (2009).

${ }^{40}$ L. Mandel, Physica scripta 1986, 34 (1986).

${ }^{41}$ L. Davidovich, Reviews of Modern Physics 68, 127 (1996)

${ }^{42}$ A. Van der Lee, N. Van Druten, M. Van Exter, J. Woerdman, J.-P. Poizat, and P. Grangier, Physical review letters 85, 4711 (2000).

${ }^{43}$ R. Boddeda, Q. Glorieux, A. Bramati, and S. Pigeon, Journal of Physics B: Atomic, Molecular and Optical Physics 52, 215401 (2019).

${ }^{44} \mathrm{M}$. Kira and S. W. Koch, Semiconductor quantum optics (Cambridge University Press, 2011)

${ }^{45}$ J. Fricke, Annals of Physics 252, 479 (1996)

${ }^{46}$ C. Gies, J. Wiersig, M. Lorke, and F. Jahnke, Physical Review A 75, 013803 (2007).

${ }^{47}$ W. W. Chow, F. Jahnke, and C. Gies, Light: Science \& Applications 3, e201 (2014).

${ }^{48}$ S. Kreinberg, W. W. Chow, J. Wolters, C. Schneider, C. Gies, F. Jahnke, S. Höfling, M. Kamp, and S. Reitzenstein, Light: Science \& Applications 6, e17030 (2017)

${ }^{49}$ J. Mork and G. Lippi, Applied Physics Letters 112, 141103 (2018). ${ }^{50}$ K. Zelaya, S. Dey, and V. Hussin, Physics Letters A 382, 3369 (2018).

${ }^{51}$ Y. Yamamoto and S. Machida, Physical Review A 35, 5114 (1987).

${ }^{52}$ S. Machida, Y. Yamamoto, and Y. Itaya, Physical review letters 58, 1000 (1987) 


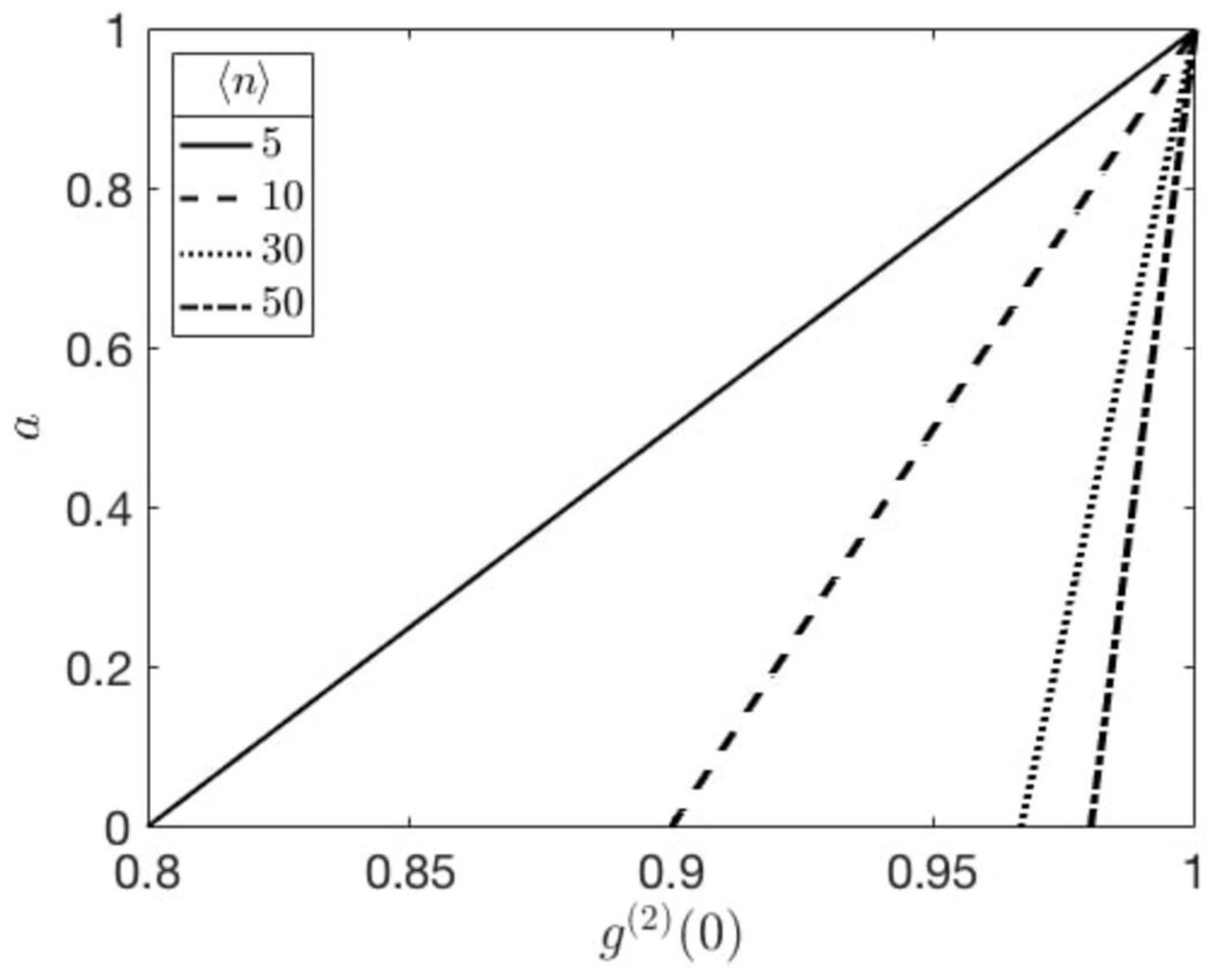

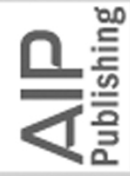




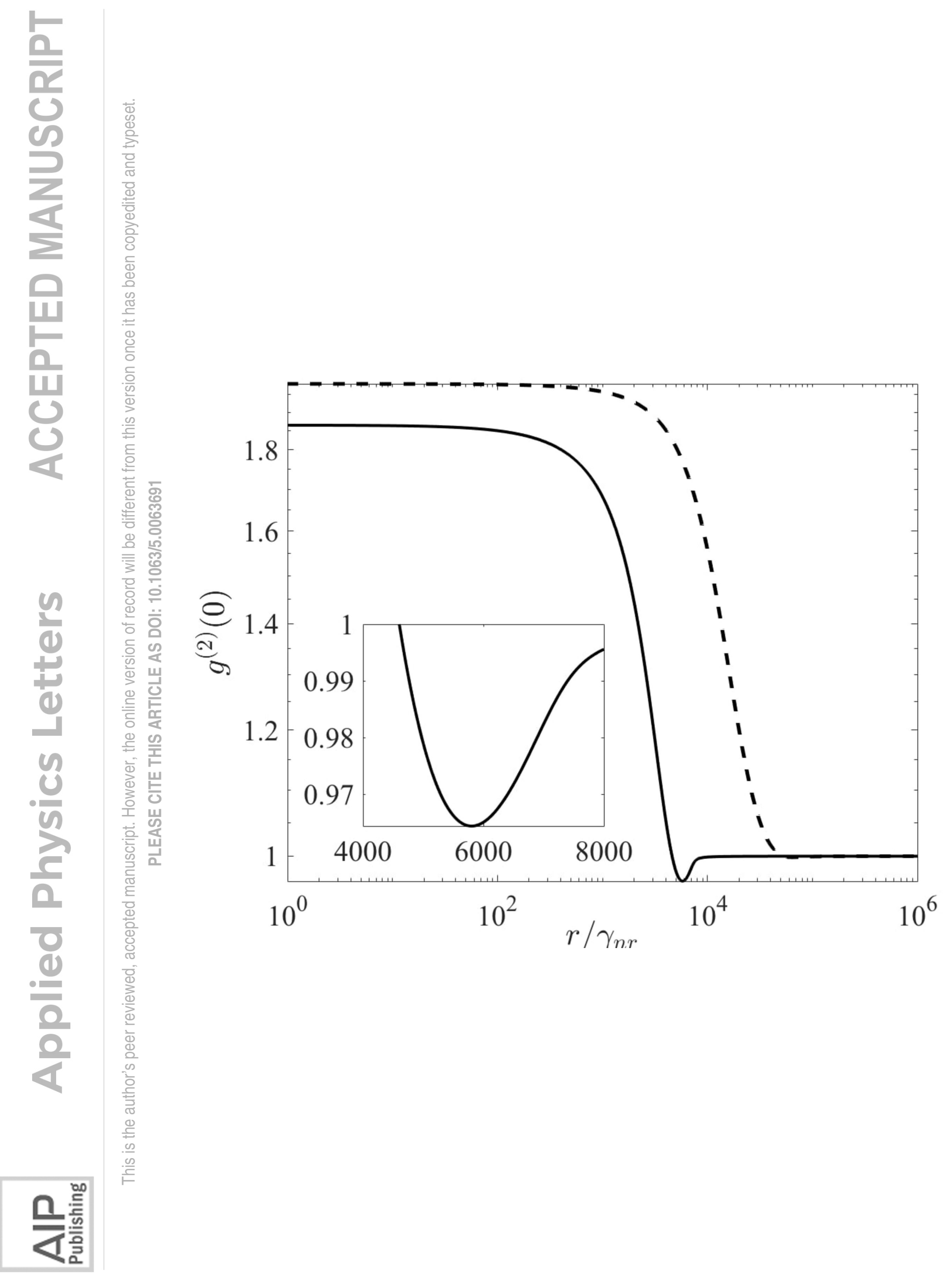




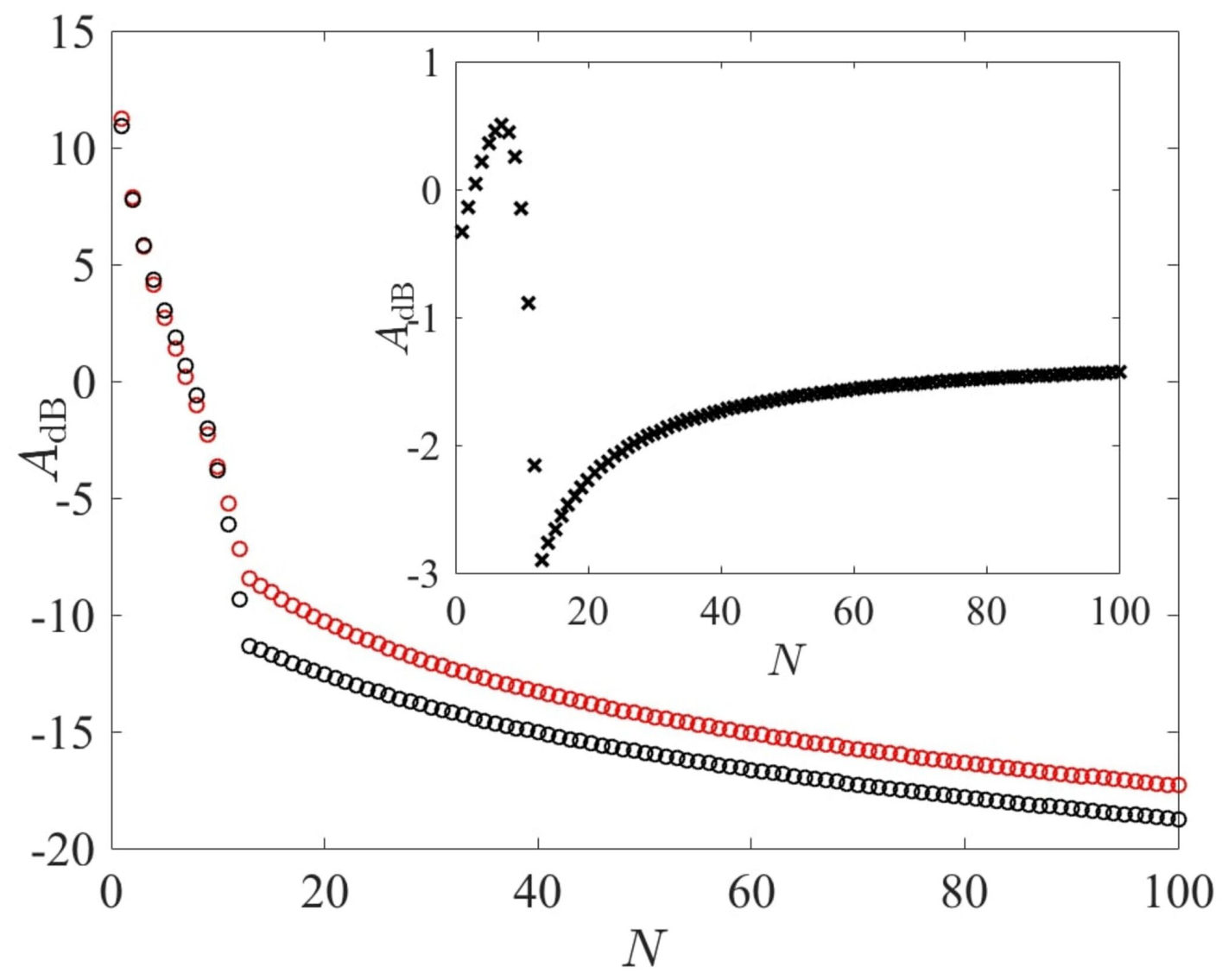




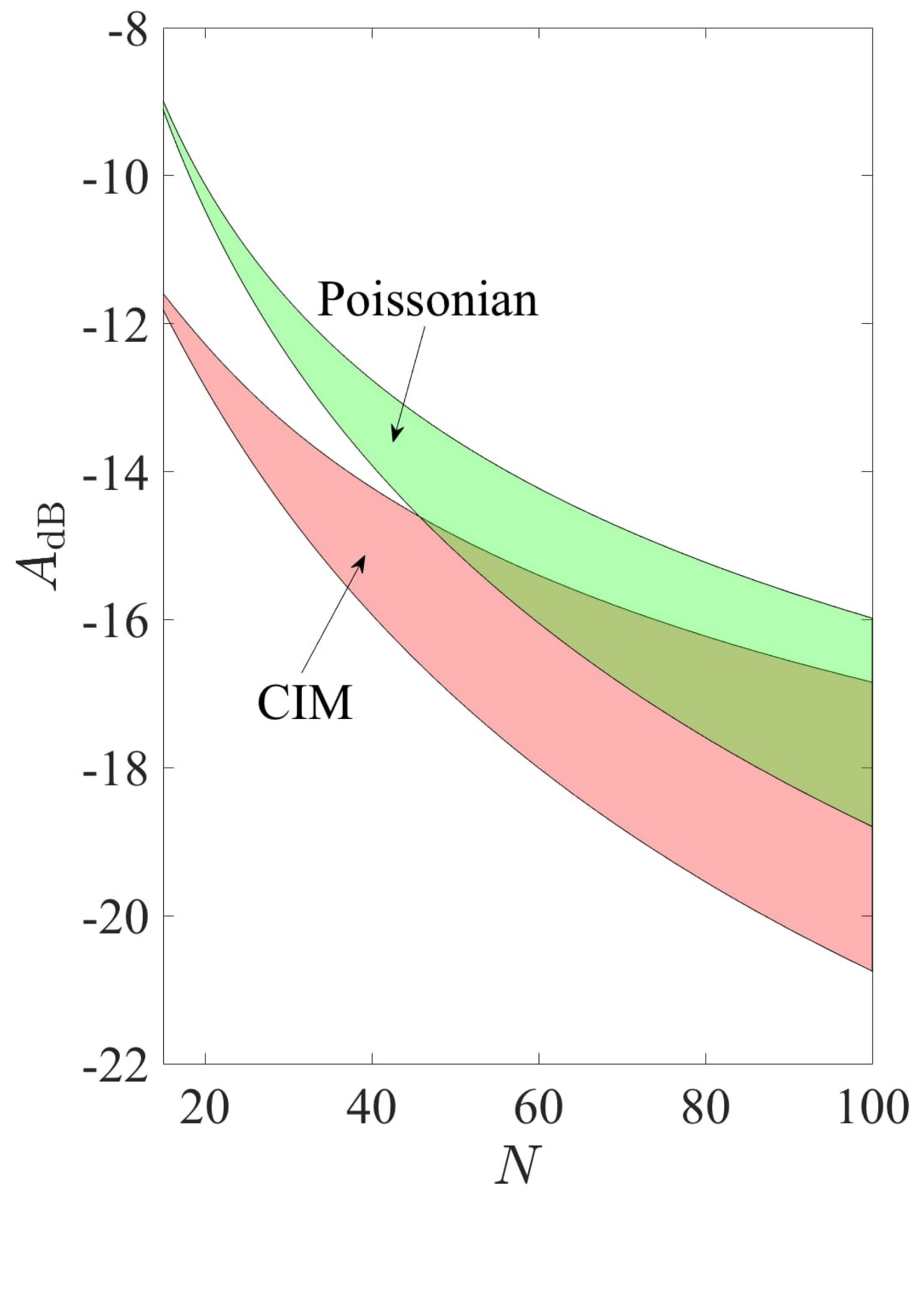




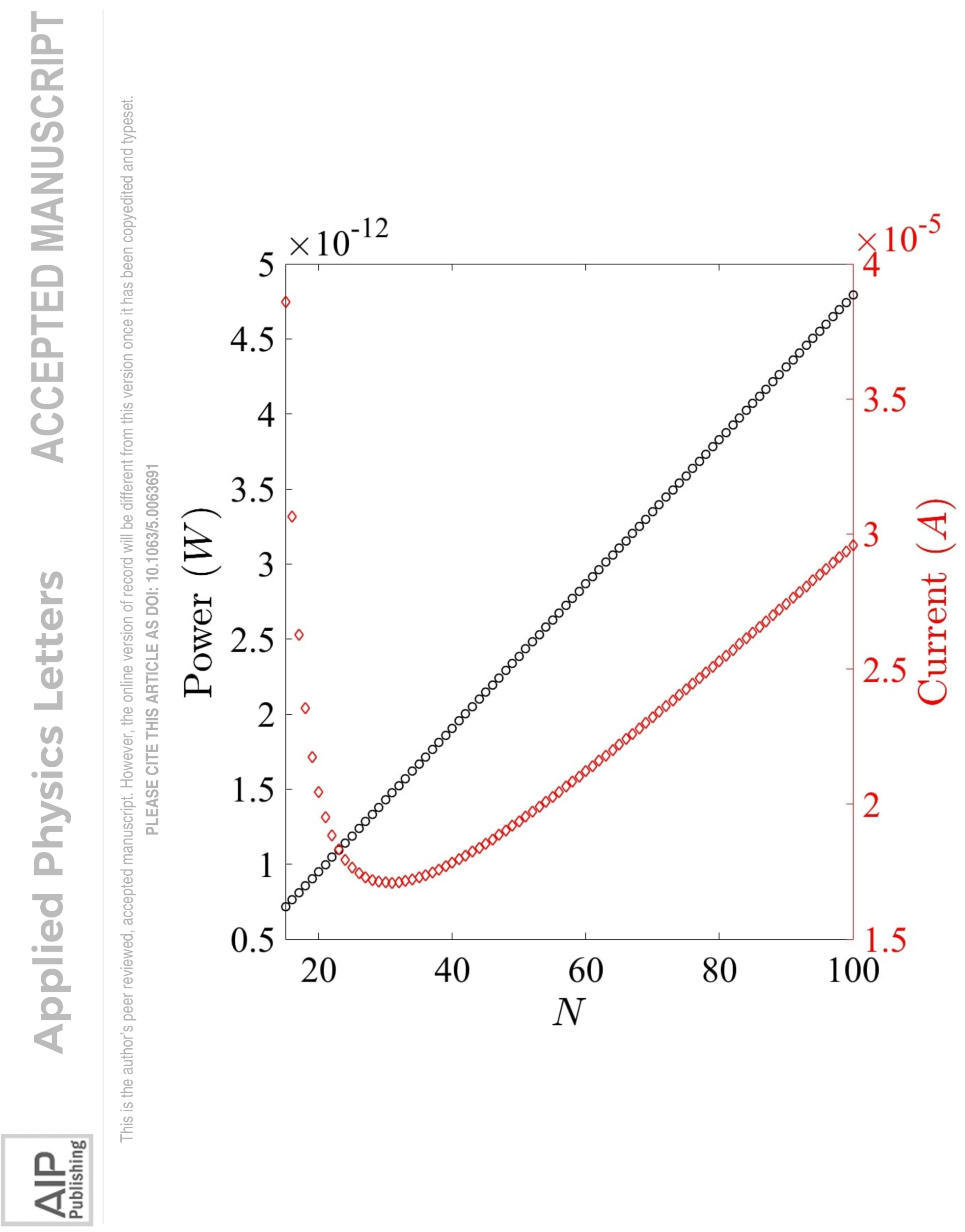

